\title{
Spectrophotometry of ring nebulæ around Wolf-Rayet stars*)
}

\author{
Manfred W. Pakull \\ Observatoire de Besançon, \\ 25044 Besançon Cedex, France
}

\begin{abstract}
Long-slit spectrophotometric observations of HII regions around Wolf-Rayet stars in the Galaxy, the Magellanic Clouds and in IC1613 are employed to measure the ionizing radiation from these stars. The sample includes all known WR stars in the SMC and 14 regions in the LMC comprising most of the known ring nebulæ. Apart from previously known HeII $\lambda 4686$ emitting nebulæ around a few WO stars, HeIII regions were discovered within the nebulæ $\mathrm{N} 76$ and N79W which are being photoionized by the strong $\mathrm{He}^{+}$Lyman continuum emission from the MagellanicWNE stars $\mathrm{AB} 7$ and $\mathrm{Br}$, respectively. The Zanstra blackbody temperatures and luminosities $(80000,95000 \mathrm{~K}$ and $\log \mathrm{L} / \mathrm{LO}_{\mathrm{O}}=5.4,6.0$, respectively) agree remarkably well with more elaborate WN model calculations and suggest a much higher bolometric correction $(\mathrm{BC}=-5.8 \mathrm{mag})$ than generally admitted for WN stars. The nebular $\lambda 4686$ fluxes are several times higher than the corresponding broad stellar features suggesting that the highly ionized gas would remain detectable at much larger distances (i.e. in HII galaxies) than the underlying hot WNE population.
\end{abstract}

\section{INTRODUCTION}

The presence of highly-ionized HII regions in Local Group galaxies and the detection of narrow HeII $\lambda 4686$ emission lines in X-ray ionized nebulæ (Pakull \& Angebault, 1986; Pakull \& Motch, 1989a,b) and many HII galaxies (i.e. Campbell, 19 1.8 ) has motivated us to systematically study the EUV emission of advanced stages of massive stellar evolution. Broad HeII and CIV emission lines in the integrated spectra of many giant HII regions and HII galaxies have revealed large populations of Wolf-Rayet stars. However, the question of whether or not WR stars do also contribute substantially to the ionizing radiation, in particular at photon energies beyond the $\mathrm{He}^{+}$Lyman edge ( $>4 \mathrm{Ryd}$, hereafter EUV) has remained open since adequate WR model atmospheres have become available only recently. On the other hand, it has been suggested that massive stars in very advanced evolutionary stages (early WC's and WO's) might not only be very luminous, but also extremely hot, reaching "effective" temperatures $>100000 \mathrm{~K}$ (see c.f. Maeder \& Meynet, 1987) that are comparable to those of the nuclei of highly ionized planetary nebulæ (PN). Accordingly, such stars might be expected to photoionize large HII regions to PNlike excitation (i.e. presence of $\mathrm{He}^{++}$ions which are not observed in normal HII regions around $\mathrm{O}$ stars). This consideration forms also the basis for the Terlevich and Melnick (1985) hypothesis, namely that WO stars ("warmers") might be responsible for the high nebular ionization in at least some types of active galaxies. However, this view has not been widely accepted, not least because sufficently high WO star EUV luminosities had not

\footnotetext{
*) Based on observations collected at the European Southern Observatory, La Silla, Chile, and at the Observatoire de Haute-Provence, France
} 
been firmly established. Moreover, the nebular $\lambda 4686$ recombination radiation should then be accompanied by even stronger broad HeII+CIV $\lambda \lambda 4600-4700$ emission generated in the stellar winds; this is generally not observed (Pakull \& Motch, 1989b).

In this paper I adress the question of ionizing radiation from WR stars and hence, their temperatures and luminosities in a more systematic fashion. The work is based on recent observations of the ionization structure of diffuse or swept-up interstellar gas around WR stars which, in many cases, assumes the morphology of a ring-like structure (ring nebulæ; cf. review by Chu in this volume). Here, I report on the results of long-slit spectroscopic observations of several Magellanic and IC1613 Wolf-Rayet stars and the surrounding interstellar medium that provide crucial informations on the EUV emission of these stars.

\section{LONG SLIT SPECTROPHOTOMETRY OF WR STARS}

\subsection{THE SAMPLE}

The sample of Wolf-Rayet stars (c.f. Table 1) was selected on the basis of: (i) presence of ring nebulæ, (ii) associated diffuse nebulosities, and after it turned out that certain WR types are particularly likely to be strong EUV emitters, (iii) WNE and WO subtypes.

TABLE 1: Sample of WR stars / nebulæ*) observed in the present study

\begin{tabular}{|c|c|c|c|c|}
\hline star & type & nebula & $5007 / \beta$ & $\lambda 4686 / \beta$ \\
\hline $\mathrm{AB} 1$ & WN 3 + O4: & (RN ?) & 5 & $<0.5$ \\
\hline $\mathrm{AB} 2$ & WN $4.5 /$ Of & (RN ?) & $3-5$ & \\
\hline $\mathrm{AB} 3$ & WN 3 + O4: & - & 2 & $<0.3$ \\
\hline $\mathrm{AB} 4$ & WN 4.5 & - & 1 & \\
\hline $\mathrm{AB} 5$ & WN 4 + O7I: & N66 & $6-7$ & \\
\hline $\mathrm{AB} 6$ & WN 3: + O6.5I: & - & & \\
\hline $\mathrm{AB} 7$ & WN 1 + O6IIIf & N76 (RN !) & $6-7$ & 0.3 \\
\hline $\mathrm{AB} 8$ & WO $4+\mathrm{O} 4 \mathrm{~V}$ & $(-)$ & & \\
\hline Br 2 & WN 1 & N79W (RN !) & $6-10$ & 0.7 \\
\hline $\operatorname{Br} 4$ & WN 1(?) & N83 (?) & 3 & $<0.04$ \\
\hline Br 12 & WN 3 & $\mathrm{~N} 16 \mathrm{~A}(\mathrm{RN})$ & 6 & $<0.03$ \\
\hline $\operatorname{Br} 16$ & WN 2.5 & $\mathrm{~N} 105$ & $4-5$ & $<0.03$ \\
\hline $\operatorname{Br} 25$ & WN 3 & N44C (RN !) & 6 & $<0.02$ \\
\hline $\mathrm{Br} 26$ & WN 7 & N198 (RN) & 3 & \\
\hline $\operatorname{Br} 29$ & WN 3/WCE & N138 (RN) & $6-8$ & \\
\hline $\operatorname{Br} 48$ & $\mathrm{WN} 4+\mathrm{OB}$ & N57C (RN) & & \\
\hline $\operatorname{Br} 51$ & WN 3 & N62 (RN !) & $9-11$ & $<0.03$ \\
\hline $\operatorname{Br} 52$ & WN 4 & N56 (RN) & $6-8$ & $<0.03$ \\
\hline $\operatorname{Br} 65 a$ & WN5 + abs & N59B & 1 & $<0.004$ \\
\hline $\mathrm{Br} 66$ & WN 3 (+WN3 ?) & N157 & 4 & $<0.007$ \\
\hline $\mathrm{Br} 93$ & WO 4 & N157 (RN !) & $4-7$ & $<0.07$ \\
\hline Br 100 & WN 4 & N74 (RN) & & \\
\hline $\mathrm{IC} 1613$ & WO & IC1613\#3(RN) & 5 & 0.3 \\
\hline
\end{tabular}

*) RN- ring nebulæ; RN! denote Magellanic ring-nebulæ not included in the compilation of Chu \& Lasker (1980) 
Special emphasis was put on Magellanic Wolf-Rayet stars, not least because the projected spectrographic slit lengths $(\approx 3$ arc min on the CCD detectors of the 2.2 and $1.5 \mathrm{~m}$ ESO telescopes) cover most of the extent of known ring nebulæ in these galaxies. Moreover, the small interstellar extinction towards, and within, the Clouds favors the detection of weak nebular emission lines since intensity is independent of distance as long as the regions are spatially resolved. Table 1 lists the observed WR stars and spectral types together with the associated nebulæ (if present) and diagnostic emission line ratios for most of them. Note that in many cases the "excitation parameter" $\mathrm{I}(\lambda 5007) / \mathrm{I}\left(\mathrm{H}_{\beta}\right)$ turns out to be extremely high $(>6)$ indicating that the ionizing WR stars are probably at least as hot as the earliest $O$ stars.

Three stars in the sample emit sufficiently strong EUV continua to excite nebular HeII $\lambda 4686$ recombination radiation in the nebulæ. They are the WO star in IC $1613 \# 3$ (Davidson \& Kinman, 1982; Pakull \& Motch, 1989b), and the WNE stars AB7 and Br2 in the SMC and LMC, respectively (Pakull et al. 1990; Pakull \& Bianchi, this volume; Niemela, this volume). To this list, WR 102 (WO2; Dopita et al. 1990; Dopita \& Lozinskaya, this volume) and $\mathrm{Br} 40 \mathrm{a}$ (WNE; Niemela, this volume) must be added. The absence of $\lambda 4686$ nebular emission in the ring nebula around $\mathrm{Br} 93$ which was independently discovered also by Heydari-Malayeri \& Melnik (this volume) implies that WO stars are not necessarily strong EUV sources; in the case of the SMC WO star AB 8 the situation is less clear since there probably is no nearby interstellar gas which could "reprocess" the EUV radiation into observable line emission.

\subsection{THE EARLIEST WN STARS: WN1}

A continuation of WNE types towards high excitation of the stellar emission lines has recently been proposed by Conti and Massey (1989) who introduced the WN1 classification for the galactic stars WR2 and the LMC member Br4 on the basis of undetectable NV ( $\left.3 \mathrm{p}^{2} \mathrm{P}^{\circ}-3 \mathrm{~s}{ }^{2} \mathrm{~S}\right) \lambda \lambda 4603,19$ emission, whereas the UV resonance NV (2p $2 \mathrm{P}^{\circ}$ $2{ }^{2}$ S) $\lambda \lambda 1238,40$ doublet remains strong. In $\mathrm{AB} 7$ and $\mathrm{Br} 2$, the ratios between the NV $\lambda \lambda 4609,19$ equivalent width (EW) and the HeII $\lambda 4686 \mathrm{EW},(\mathrm{NV} / \mathrm{HeII})$, are $<0.02$ and 0.04 , respectively, both values being significantly smaller than in the galactic and LMC stars which Conti and Massey classify as WN2, or later. The (NV/HeII) ratio for the latter stars are all $>0.1$, suggesting that we might draw a dividing line between WN1 and the later WN subtypes, according to whether (NV/HeII) is smaller or larger than about 0.07 .

It is of some interest to note that whereas the $\lambda \lambda 4603,19$ lines become weaker towards earlier WN spectral types, the largely recombination fed NV(7-6) subordinate $\lambda \lambda 4933,45$ doublet (Hillier, 1988) remains comparatively strong. The latter emission lines are not part of the classical classification scheme; however, my CCD spectra of the galactic WN1 "standard" WR2 clearly display this emission at about the same strength as in $\mathrm{Br} 2$ $(\mathrm{EW} \approx 10 \AA$ ).

\subsection{AB7 AND BR 2}

These WN1 stars do completely photoionionize helium in the inner $\sim 20$ pc of their ring nebulæ. The electron temperatures, $\mathrm{T}_{\mathrm{e}}(\mathrm{OIII}) \approx 16000 \mathrm{~K}$, in both the nebulæ around $\mathrm{AB} 7$ (N76) and around $\mathrm{Br} 2$ (N79W) indeed strongly suggest that photoionization rather than ionization by shocks is the dominant process that feeds the nebular HeII recombination radiation. These results have several interesting consequences. First of all, the stellar wind layers in which the EUV radiation is formed must be sufficiently hot to strongly emit in the $\mathrm{He}^{+}$-Lyman continuum. Moreover, the outer wind zones have to be optically thin in the EUV, i.e. helium should be completely ionized throughout the wind (see below). Then, using the photon-counting property of the nebular $\lambda 4686$ recombination line one can derive the rate of $\mathrm{He}^{+}$-ionizing photons (Q4; i.e. $\mathrm{h} v>4$ Ryd) escaping from the Wolf-Rayet wind. 
Using the beautifully simple, but nevertheless extremely powerful Zanstra method which compares nebular $\lambda 4686$ flux to the stellar flux at observable wavelengths yields blackbody temperatures of 80000 and $95000 \mathrm{~K}$, and luminosities, $\log \mathrm{L} / \mathrm{L}_{\mathrm{O}}=5.3$ and 6.0 , for these Magellanic WN1 stars (see Table 2). The analysis is rather straightforeward for the single star $\mathrm{Br} 2$; in the case of the WR+O6f binary AB7, the visual magnitude of the WN1 component was estimated by subtracting from the binary spectrum appropriately scaled spectra of a single $\mathrm{O} 6$ star until the the $\mathrm{H}, \mathrm{HeI}$ and $\mathrm{HeII}$ absorption lines disappeared (Pakull et al. 1990).

One might argue that the extremely high $\mathrm{HeII} \lambda 4686 / \mathrm{H}_{\beta}$ line ratio $(=0.7$ !) in the brightest parts of the ring nebula $\mathrm{N} 79 \mathrm{~W}$ implies a significantly harder ionizing spectrum than a $95000 \mathrm{~K}$ blackbody. For example, from their analysis of nebular lines $\left(\lambda 4686 / \mathrm{H}_{\beta}=\right.$ 0.55) around the WO star WR102, Dopita et al. (1990, and this volume) derive an ionizing temperature $\mathrm{T}_{\mathrm{BB}} \approx 150000 \mathrm{~K}$. Moreover, Stasinska \& Tylenda (1986) have pointed out that this line ratio levels off at $\mathrm{T}_{\mathrm{BB}}>250000 \mathrm{~K}$ and never exceeds a value of about 0.6 . However, it should be kept in mind that the observed ratio in $\mathrm{N} 79 \mathrm{~W}$ mainly refers to the $\mathrm{He}^{++}$zone within a larger (ill defined) HII region rather than to the total emission calculated in the model nebulæ. Another effect increasing the $\lambda 4686 / \mathrm{H}_{\beta}$ ratio is the possibility that $\mathrm{N} 79 \mathrm{~W}$ might well be partially transparent to the $\mathrm{H}$-ionizing photons but optically thick in the $\mathrm{He}^{+}$ Lyman continuum (EUV).

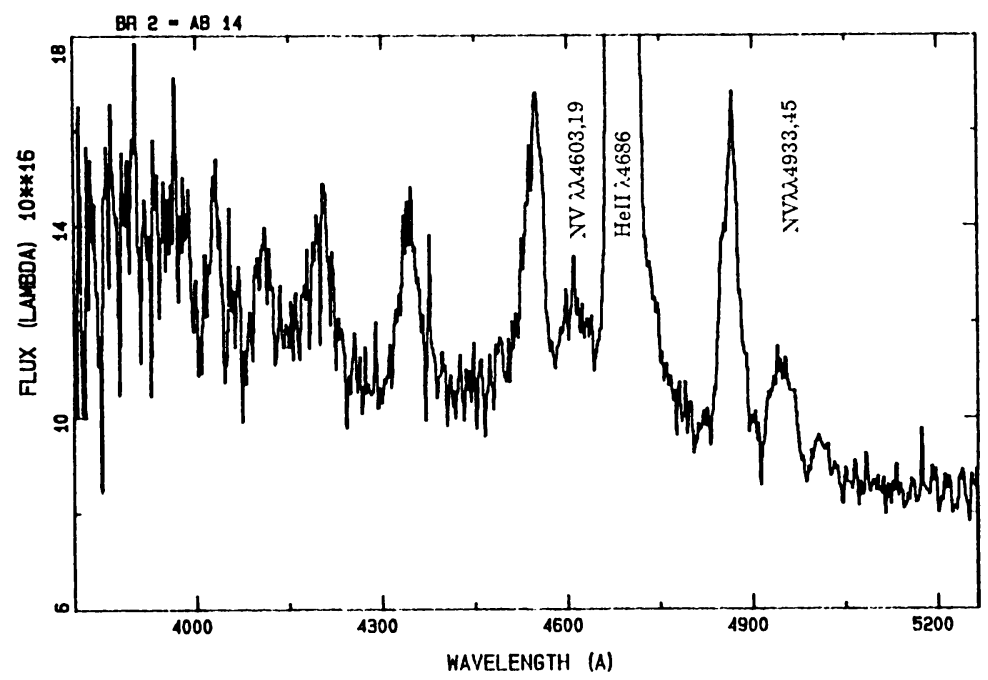

FIG. 1: Blue CCD spectrum of the WN1 star Br2. Note the presence of $\lambda \lambda 4933,45$ emission $(\mathrm{EW} \approx 9 \AA$ ) and the very weak $\mathrm{NV} \lambda \lambda 4603,19$ doublet $(\mathrm{EW} \approx 6 \AA$ ). The equivalent width of the strong HeII $\lambda 4686$ line is $160 \AA$.

\section{COMPARISON WITH WR MODEL SPECTRA}

The condition that the (helium) winds of AB7 and Br2 must be completely ionized allows to draw an important lower limit on the rate of $\mathrm{He}^{+}$ionizing photons, $\mathrm{Q}_{4}$, emitted by WR "cores". Assuming for simplicity a constant wind velocity law, $v(R)=v_{\infty}$, and furthermore provided that photoionization from the ground state is the dominant process for 
the He ionization beyond the radius REUV where the EUV continuum is formed, this translates to:

$\mathrm{Q}_{4}>110^{49} \mathrm{~s}^{-1}\left[\mathrm{M}_{-4} / \mathrm{v}_{2500}\right]^{2}\left[\mathrm{R}_{\mathrm{EUV}} / \mathrm{RO}_{\mathrm{O}}\right]^{-1}$,

where an electron temperature of $80000 \mathrm{~K}$ has been assumed, M-4 is the mass loss rate in units of $10^{-4} \mathrm{MO} / \mathrm{y}$, and $\mathrm{v}_{2} 500=\mathrm{v}_{\infty} /(2500 \mathrm{~km} / \mathrm{s})$. This order-of-magnitude estimate suggests that even very hot WR "cores" cannot ionize the wind completely, once the mass loss-rates become sufficiently high. On the other hand, the relatively low $\mathrm{M} / \mathrm{v}_{\infty}$ ratios together with the lower limit of the temperature parameter, $T *$, which were derived from the optical and UV spectra (see below) already strongly favour the escape of EUV radiation from $\mathrm{AB} 7$ and $\mathrm{Br} 2$ beyond the stellar wind.

A remarkable result of this simple analysis is that the (pure helium) WR model spectra calculated by the Kiel group (Schmutz, Hamann \& Wessolowsky, 1989 and references therein; Schmutz, these proceedings; Hamann, these proceedings) agree rather well with the observed stellar $\mathrm{He}$ emission line strenghs and absolute magnitudes of $A B 7$ and $\mathrm{Br} 2$, if Zanstra blackbody temperatures are identified with the effective temperatures, $\mathrm{T*}$, at the "core radius", $\mathrm{R*}$, which is the inner boundary of the model atmospheres. According to these models, emission lines and continuum emission of WN stars are largely determined by $T *$ and the radius parameter, $R *\left[M_{-4} / \mathrm{v}_{2500}\right]^{-2 / 3}$.

TABLE 2: WN1 star parameters

\begin{tabular}{|c|c|c|c|c|c|c|c|c|c|}
\hline Object & $\mathrm{MV}_{\mathrm{V}}$ & $\mathrm{BC}$ & $\begin{array}{c}\mathrm{Q}_{4} \\
{\left[\mathrm{~S}^{-1}\right]}\end{array}$ & $\begin{array}{c}\mathrm{v}_{\infty} \\
{[\mathrm{km} / \mathrm{s}\rceil}\end{array}$ & $\begin{array}{r}\text { TBB } \\
{[\mathrm{kK}]}\end{array}$ & $\begin{array}{l}\mathrm{L}_{\mathrm{BB}} \\
\mathrm{LO}]\end{array}$ & $\begin{array}{l}\mathrm{T} * \\
\mathrm{kK}\end{array}$ & $\begin{array}{l}\mathrm{L}_{\text {model }} \\
\text { Lol }\end{array}$ & $\begin{array}{c}\mathrm{M} \\
10^{-4} \underline{\mathrm{M}} \mathrm{O} / \mathrm{y}\end{array}$ \\
\hline $\mathrm{AB} 7$ & -4.4 & -5.9 & $1.510^{48}$ & 1600 & 80 & $910^{5}$ & 75 & $110^{6}$ & 0.14 \\
\hline $\mathrm{Br} 2$ & -2.6 & -5.7 & $810^{47}$ & 2500 & 95 & $210^{5}$ & 90 & $210^{5}$ & 0.15 \\
\hline
\end{tabular}

The non-detection of HeI features in WNE stars have so far only allowed to place lower limits on $\mathrm{T} *$. Now, the observation of nebular $\lambda 4686$ emission around some of these stars opens the possiblity to substantially narrow down the allowed range of parameters for a given Wolf-Rayet star. After this conference, Werner Schmutz (1990b) has kindly informed me of his extended model calculations concerning the continuum emission down to wavelengths of $100 \AA$. At least in the parameter range covered by this work the validity of the EUV emitter criterion mentioned earlier is nicely confirmed. In Table 2 the relevant Kiel-model stellar parameters for $\mathrm{AB} 7$ and $\mathrm{Br} 2$ are summarized together with the results based on naïve blackbody fits. The advocates of a WN "universal" bolometric correction of - 4.5 might in particular note that both $\mathrm{BB}$ and more sophisticated modeling of these WN1 stars imply BC $<-5.5$.

\section{CONCLUSIONS}

The main result of the present study concerns the discovery of strong EUV radiation from some WNE stars in the Magellanic Clouds. This constitutes the first direct proof of their significantly higher effective temperatures as compared to the $O$ star progenitors. Although the EUV emitters $\mathrm{AB7}$ and $\mathrm{Br} 40 \mathrm{a}$ are in binary systems, the lack of a luminous companion of $\mathrm{Br} 2$ suggests that also single star evolution might lead to $\approx 100000 \mathrm{~K}$ WN-type remnants. 
The discovery of strong HeII $\lambda 4686$ recombination radiation from the WNE ring nebulæ N76 and N79W also suggests a natural explanation for the previously puzzling presence of this line in a number of giant HII regions and HII galaxies in terms of a population of such WNE stars. In fact, one might call these objects genuine warmers since the EUV fed $\lambda 4686$ emission turned out to be about 8 times stronger than the corresponding broader stellar feature. These warmers will not be detectable any more in distant galaxies except for their ionizing radiation.

\section{REFERENCES}

Campbell, A. 1988, Astrophys. J. 335, 644

Chu, Y.-H. \& Lasker, B.M. 1980, Pub. A. S. P. 92,730

Conti, P.S. \& Massey, P. 1989, Astrophys. J. 337, 251

Davidson, K. \& Kinman, T.D. 1982, Pub. A. S. P. 94, 634

Dopita, M.A. \& Lozinskaya, T.A. 1990, in this volume

Dopita, M.A., Lozinskaya, T.A., McGregor, P.J. \& Rawlings, S.J. 1990, Astrophys. J. 351, 563

Hamann, W.-R. 1990, in this volume

Heydari-Malayeri, M. \& Melnick, J. 1990, in this volume

Hillier, D.J. 1988, Astrophys. J. 327, 822

Maeder, A. \& Meynet, G. 1987, Astron. Astrophys. 182, 245

Niemela, V.S. 1990 , in this volume

Pakull, M.W. \& Angebault, L.P. 1986, Nature 322, 511

Pakull, M.W. \& Motch, 1989a, Nature 337, 337

Pakull, M.W. \& Motch, C. 1989b, ESO workshop on 'Extranuclear Activity in Galaxies', E.J.A. Meurs \& R.AE. Fosbury (eds.) p 285

Pakull, M.W., Testor, G. \& Bianchi, L. 1990, submitted to Astron. Astrophys.

Pakull, M.W. \& Bianchi, L. 1990, in this volume

Schmutz, W., Hamann, W.-R. \& Wessolowsky, U. 1989, Astron. Astrophys. 210, 236

Schmutz, W. 1990a, in this volume

Schmutz, W. $1990 \mathrm{~b}$, private communication

Stasinka, G. \& Tylenda, R. 1986, Astron. Astrophys. 155, 137

Terlevich, R. \& Melnick, J. 1985, Mon. Not. R. astr. Soc. 213, 841

\section{DISCUSSION}

Cassinelli: Most impressive stars. Radiation pressure would not have any trouble driving their winds. Does this violate the newer picture for evolution?

Langer: You would not really conclude that these positions fit very well to the tracks. However, after the core helium exhaustion, the tracks are nearly predicted to lead to very high effective temperatures and also to somewhat higher luminosities again. So, this picture would really mean that these warmers correspond to the latest evolutionary stage, carbon burning or something like that which would also account for the small number of those objects.

Pakull: These stars are WN's, not WC's.

Langer: But still, you have the possibility of some WN stars being treated as supernova candidates too, so, you must not lead all WR stars to the WC stage. 